\title{
Mejoras en el área de atención a clientes corporativos de una empresa de telecomunicaciones
}

\begin{abstract}
RESUMEN
En esta investigación se ha evaluado la situación actual del área de atención de reclamos hechos por clientes corporativos en una empresa de telecomunicaciones. Se utilizó el software Arena para realizar simulaciones, con el objetivo de obtener soluciones que mejoren los indicadores de gestión. Se propusieron tres alternativas de solución que fueron evaluadas económicamente, obteniéndose como mejor opción, aquella que recomienda la asignación de cinco ejecutivos a tiempo completo para la atención de clientes en el área de Back Office en lugar de ocho ejecutivos a medio tiempo; asimismo, se recomienda la asignación de 43 ejecutivos a tiempo completo para la atención de clientes en el área de Front Office, es decir, dos ejecutivos menos.
\end{abstract}

Palabras clave: Simulación, empresa de telecomunicaciones, cliente corporativo.

\section{IMPROVEMENTS FOR THE} CORPORATIVE CLIENTS OF THE CUSTOMER SERVICES DEPARTMENT OF A TELECOMMUNICATION COMPANY

ABSTRACT

The current situation of the corporative customers' complaints of the Customer Services Department of a telecommunication company has been evaluated in this research. Software Arena has been used to perform simulations to get solutions that will improve the management rates of the Customer Services Department. Three solutions were proposed. However after evaluating each of them, the one that considers having five full time executives at the Back Office Area, instead of eight part time executives was considered the best alternative to choose. Additionally, it is recommended to have forty three full time executives at the Front Office area. This decision considers diminishing the personnel of this area in two executives.

Key words: Simulation, telecomunication company, corporative customer.

\section{INTRODUCCIÓN}

Las empresas de servicios brindan a sus clientes diversos productos con valor agregado que les permita diferenciarse de sus competidores, sin embargo también es importante que la calidad del servicio, entendida como el nivel de satisfacción del cliente, sea muy satisfactoria. El nivel de satisfacción del cliente es consecuencia de la interacción del personal de contacto de la empresa, en las áreas de atención al cliente, con los clientes en general. La empresa de telecomunicaciones que se estudiará, es una de las más importantes del país, y ofrece una variedad de equipos de telefonía celular, así como servicios complementarios para estos equipos.

En la empresa de telecomunicaciones en estudio, el área de reclamos y variaciones es una de las más críticas debido a que la atención oportuna y eficiente de los clientes influirá en la fidelidad de estos con la empresa. Un reclamo es consecuencia de problemas con los equipos y los servicios posventa. Una variación es cualquier tipo de pedido solicitado por el cliente (por ejemplo, cambio en el plan tarifario, cancelación de línea, detalle de llamadas, envío de recibo por correo electrónico, duplicado de recibo, facturación detallada, etc.).

El área de reclamos y variaciones atiende a clientes corporativos y cuenta con un grupo de ejecutivos que realiza las tareas de Front Office (área de atención telefónica de los clientes) y de Back Office (área de resolución de pedidos de los clientes).

Dada la importancia que en el área de reclamos y variaciones, se brinde a los clientes una atención rápida y satisfactoria, el estudio a realizar evaluará las zonas Front Office y Back Office para proponer soluciones que aumenten el nivel de satisfacción de los clientes.

Un indicador de gestión que se emplea en la empresa es el indicador de nivel de servicio. Este indicador brinda información acerca del porcentaje de casos que son resueltos en un tiempo preestablecido como tiempo objetivo. Otro indicador gestión es el nivel de producción. Este indicador es utilizado para analizar qué tan efectivo es cada uno de los ejecutivos y mide la cantidad promedio de casos que cada ejecutivo resuelve al día.

\footnotetext{
Ingeniera Industrial, PUCP, Analista, Banco de Crédito del Perú. E-mail: mbrousset@pucp.edu.pe

2 Doctor en Ingeniería Industrial, UNMSM, Profesor del Departamento de Ingeniería, PUCP. E-mail: miguel.mejia@pucp.edu.pe
} 


\section{DESCRIPCIÓN DEL SISTEMA ACTUAL}

El cliente se comunica con la empresa de telecomunicaciones que le brinda servicios de telefonía a través de teléfono, correo electrónico, fax o carta. El medio más utilizado es el teléfono por ello el estudio se centrará en el Centro de Llamadas. El horario de atención del Centro de Llamadas es de lunes a sábado, ocho horas diarias.

Según el tipo de cliente que se haya comunicado, su caso es atendido por grupos predeterminados de atención en el Front Office usando los siguientes criterios:

- Si llama la persona de contacto de una empresa que pertenece al tipo de cliente "Grandes Cuentas", ésta es atendida por un ejecutivo del grupo Grandes Cuentas.

- Si llama cualquier empleado de una empresa que pertenece al tipo de cliente "Grandes Cuentas" éste es atendido por un ejecutivo del grupo Front Office Corporativo.

- Si llama cualquier empleado de una empresa que pertenece al tipo de cliente "Corporativo y PYMES", éste es atendido por un ejecutivo del grupo Front Office Corporativo.

Los ejecutivos que atienden en el Front Office Corporativo son 65 personas, distribuidas de la siguiente manera:

- Grandes Cuentas: 20 empleados, que atienden a los clientes durante ocho horas diariamente.

- Front Office Corporativo: 45 empleados, que atienden a los clientes durante seis horas diariamente.

Los ejecutivos del Front Office Corporativo tratan de resolver los casos inmediatamente. De presentarse un caso que requiera mayor tiempo para ser resuelto, este será derivada a los ejecutivos del Back Office Corporativo según los siguientes criterios:

- Cualquier caso presentado por un empleado, sea la persona de contacto o no, de una empresa que pertenece al tipo de clientes "Grandes Cuentas" es atendido por un ejecutivo del grupo Grandes Cuentas.

- Un caso de facturación o variación presentado por un empleado de una empresa que pertenece al tipo de clientes "Corporativo y PYMES" es atendido por un ejecutivo del grupo Back Office Corporativo.

- Un caso de reclamo o variación presentado por un empleado de una empresa que pertenece al tipo de clientes "Corporativo y PYMES" es atendido por un ejecutivo del grupo Front Office Corporativo.

Los ejecutivos que atienden en el Back Office Corporativo son 73 personas, distribuidas de la siguiente manera:

- Grandes Cuentas: 20 empleados, que atienden a los clientes durante ocho horas diariamente. Son los mismos que se mencionan en el Front Office.

- Back Office Corporativo: 8 empleados, que atienden casos de variación durante tres horas y treinta y ocho minutos aproximadamente y casos de facturación el resto del día.

- Front Office Corporativo: 45 empleados, que atienden casos de reclamo y variación durante una hora y cuarenta y cinco minutos diariamente. Son los mismos que se mencionan en el Front Office.

La empresa busca satisfacer las necesidades de todos sus clientes, sin embargo, presta mayor atención a los clientes de "Grandes Cuentas", brindándoles atención personalizada rápida y eficiente. Para ello se cuenta con un grupo de ejecutivos denominado Grandes Cuentas que se dedica íntegramente a atender estos casos. En el estudio a realizar no se considerarán a estos clientes.

El objetivo de la empresa es resolver los casos de reclamo en menos de cuatro días para el tipo de clientes "Grandes Cuentas" y en menos de seis días para el tipo de clientes "Corporativo y PYMES". Los casos de variación se deben resolver en menos de tres días para ambos tipos de clientes. En el estudio a realizar no se consideran los casos de facturación.

Los clientes se clasifican en tres tipos que se muestran en la Tabla 1.

Tabla N.․ 1: Clasificación de los clientes atendidos en el área de reclamos y variaciones.

\begin{tabular}{|c|l|}
\hline Tipo & \multicolumn{1}{|c|}{ Descripción } \\
\hline 1 & Cliente que presenta un reclamo \\
\hline 2 & $\begin{array}{l}\text { Cliente que presenta una } \\
\text { variación }\end{array}$ \\
\hline 3 & $\begin{array}{l}\text { Cliente cuyo pedido se resuelve } \\
\text { durante la llamada }\end{array}$ \\
\hline
\end{tabular}

Fuente: Elaboración propia

La probabilidad asociada a cada tipo de cliente se muestra en la Tabla N. ${ }^{\circ} 2$. Estas probabilidades se obtuvieron de 77,716 casos atendidos por el área de reclamos y variaciones durante dos meses. 
Tabla N. ${ }^{\circ}$ 2: Probabilidad de que un cliente sea de un tipo determinado.

\begin{tabular}{|c|c|}
\hline Tipo & Probabilidad \\
\hline 1 & 0.0048 \\
\hline 2 & 0.2795 \\
\hline 3 & 0.7157 \\
\hline Total & 1.0000 \\
\hline
\end{tabular}

Fuente: Empresa de telecomunicaciones; elaboración propia

\section{MODELADO DEL SISTEMA}

Tamaño de muestra

Para cada día de la semana, se evaluó el comportamiento de los tiempos entre llegadas y la variación de éstos según el tamaño de muestra. En la Tabla 3 se muestra el tamaño de muestra para cada día en base a datos de 2 meses. La fórmula empleada para determinar el tamaño de muestra con un nivel de confiabilidad del $95 \%$ es $N=(1.96)^{*}(S)^{2} / d^{2}$

\section{Tiempos de servicio}

El tiempo promedio de atención de una interacción en el Front Office Corporativo se asume constante y dura 6.08 minutos. El tiempo promedio de atención de un reclamo y variación en el Back Office Corporativo se muestra en la Tabla N. ${ }^{\circ} 5$.

Tabla N. ${ }^{\circ}$ 5: Duración del servicio.

\begin{tabular}{|l|c|}
\hline \multicolumn{1}{|c|}{ Proceso } & $\begin{array}{c}\text { Tiempo } \\
\text { (minutos) }\end{array}$ \\
\hline Atención de reclamos & 136 \\
\hline Atención de variaciones & 15 \\
\hline
\end{tabular}

Fuente: Elaboración propia

La atención de reclamos considera varias actividades que se presentan en la Tabla N. ${ }^{\circ} 6$.

Tabla N. ${ }^{\circ}$ 3: Tamaño de muestra para cada día simulado.

\begin{tabular}{|l|c|c|c|c|}
\hline $\begin{array}{c}\text { Día de la } \\
\text { semana }\end{array}$ & Promedio & $\begin{array}{c}\text { Desviación } \\
\text { estándar }(\mathrm{S})\end{array}$ & $\begin{array}{c}\text { Precisión } \\
(\mathrm{d})\end{array}$ & $\begin{array}{c}\text { Tamaño de } \\
\text { muestra }(\mathrm{N})\end{array}$ \\
\hline Lunes & 1,810 & 173 & 29.1 & 136 \\
\hline Martes & 1,748 & 183 & 30.2 & 141 \\
\hline Miércoles & 1,758 & 350 & 51.9 & 176 \\
\hline Jueves & 1,704 & 172 & 29.0 & 135 \\
\hline Viernes & 1,354 & 324 & 49.4 & 165 \\
\hline Sábado & 735 & 204 & 34.5 & 135 \\
\hline Domingo & 330 & 90 & 17.6 & 101 \\
\hline
\end{tabular}

Fuente: Empresa de telecomunicaciones; elaboración propia

Tiempos entre llegadas

En la Tabla N. ${ }^{\circ} 4$ se presenta la distribución de probabilidades del tiempo entre llegadas para cada día de la semana. Se asume que el día tiene ocho horas pues éste es el tiempo que trabaja cada ejecutivo.

Tabla N. ${ }^{\circ}$ 4: Distribución de probabilidades del tiempo entre llegadas.

\begin{tabular}{|l|l|}
\hline \multicolumn{1}{|c|}{$\begin{array}{c}\text { Día de la } \\
\text { semana }\end{array}$} & \multicolumn{1}{c|}{ Distribución de semana } \\
\hline Lunes & EXPO $(1,810$ ocurrencias / día $)$ \\
\hline Martes & EXPO $(1,748$ ocurrencias / día $)$ \\
\hline Miércoles & EXPO (1,758 ocurrencias / día $)$ \\
\hline Jueves & EXPO (1,704 ocurrencias / día $)$ \\
\hline Viernes & EXPO (1,354 ocurrencias / día $)$ \\
\hline Sábado & EXPO (735 ocurrencias / día $)$ \\
\hline Domingo & EXPO (330 ocurrencias / día $)$ \\
\hline
\end{tabular}

Fuente: Elaboración propia
Tabla $\mathbf{N}^{\circ}$ 6: Actividades asociadas a la atención de reclamos.

\begin{tabular}{|l|c|}
\hline \multicolumn{1}{|c|}{ Actividad } & $\begin{array}{c}\text { Tiempo } \\
\text { (minutos) }\end{array}$ \\
\hline Lectura de caso & 5 \\
\hline Búsqueda de recibo & 4 \\
\hline Búsqueda de acuerdos & 6 \\
\hline Revisión de información histórico & 7 \\
\hline Consulta de detalle & 16 \\
\hline Envío de caso a cola respectiva & 6 \\
\hline Recepción de caso, análisis y cálculo & 33 \\
\hline $\begin{array}{l}\text { Registro en el módulo de gestión de } \\
\text { reclamos }\end{array}$ & 16 \\
\hline Redacción de carta resolutiva & 18 \\
\hline Realización de notas del caso & 12 \\
\hline \multicolumn{2}{|c|}{ Total } \\
\hline
\end{tabular}

Fuente: Elaboración propia 
También considera algunas incidencias que se muestran en la Tabla $\mathrm{N} .^{\circ} 7$.

Tabla N..$^{\circ}$ 7: Incidencias durante la atención de reclamos

\begin{tabular}{|l|c|}
\hline \multicolumn{1}{|c|}{ Incidencia } & $\begin{array}{c}\text { Tiempo } \\
\text { (minutos) }\end{array}$ \\
\hline $\begin{array}{l}\text { Consulta y/o asesoría a otros } \\
\text { ejecutivos }\end{array}$ & 2 \\
\hline Preguntas de CRM facturación & 7 \\
\hline $\begin{array}{l}\text { Falla o caída de los sistemas } \\
\text { informáticos }\end{array}$ & 4 \\
\hline \multicolumn{1}{|c|}{ Total } & 13 \\
\hline
\end{tabular}

Fuente: Elaboración propia

El tiempo total de atención de los diversos tipos de reclamos se ha considerado como $123+13=136$ minutos. No se considera el tiempo que el caso permanece en otras áreas administrativas (tres días).

La atención de variaciones también considera varias actividades que se presentan en la Tabla N.$^{\circ} 8$.

Tabla N.․ 8: Actividades asociadas a la atención de variaciones.

\begin{tabular}{|l|c|}
\hline \multicolumn{1}{|c|}{ Actividad } & $\begin{array}{c}\text { Tiempo } \\
\text { (minutos) }\end{array}$ \\
\hline Lectura del caso & 2 \\
\hline Análisis de la necesidad del cliente & 2 \\
\hline Verificación de datos & 1 \\
\hline Llenado de plantilla & 2 \\
\hline Ejecución de la necesidad del cliente & 3 \\
\hline Realización de notas del caso & 2 \\
\hline $\begin{array}{l}\text { Envío de respuesta al cliente por } \\
\text { correo electrónico }\end{array}$ & 3 \\
\hline \multicolumn{2}{|c|}{ Total } \\
\hline
\end{tabular}

Fuente: Elaboración propia

El estudio que se hará a continuación busca mejorar los indicadores de gestión. Se analizará el sistema actual y tres propuestas de mejora. También se hará una evaluación económica de cada caso presentado.

\section{Acerca del modelo de simulación}

En el diseño del modelo se han considerado los siguientes criterios:

- Se simulan cinco años (876,000 minutos).

- Se efectuaron cuarenta réplicas de la simulación.

- Se empleará un nivel de confiabilidad del $95 \%$ para los intervalos de confianza de los tiempos de atención y de espera.

\section{RESULTADOS DEL SISTEMA ACTUAL}

Los tiempos obtenidos en el sistema actual, indicados en intervalos de confianza, se resumen en la Tabla N. ${ }^{\circ} 9$.

Tabla N..$^{\circ}$ 9: Resumen de la situación actual para atención de reclamos y variaciones.

\begin{tabular}{|c|c|}
\hline Descripción & Intervalos de confianza \\
\hline $\begin{array}{l}\text { Área de Front Office } \\
\text { - } 45 \text { empleados } \\
\text { de Front Office } \\
\text { Corporativo atienden } \\
\text { casos de reclamo y } \\
\text { variación durante seis } \\
\text { horas diariamente. } \\
\text { Área de Back Office } \\
\text { - } 8 \text { empleados de Back } \\
\text { Office Corporativo } \\
\text { atienden casos de } \\
\text { variación durante tres } \\
\text { horas y treinta y ocho } \\
\text { minutos. } \\
\text { - } 45 \text { empleados } \\
\text { de Front Office } \\
\text { Corporativo atienden } \\
\text { casos de reclamo } \\
\text { y variación durante } \\
\text { una hora y cuarenta } \\
\text { y cinco minutos } \\
\text { diariamente. }\end{array}$ & $\begin{array}{l}\text { - }[1,390 ; 1,950] \text { minutos } \\
\text { para el tiempo total de } \\
\text { atención de reclamos. } \\
\text { - }[1,005 ; 1,494] \text { minutos } \\
\text { para el tiempo total de } \\
\text { atención de variaciones. } \\
\text { - }[0.751 ; 0.762] \text { minutos } \\
\text { para el tiempo de } \\
\text { espera en la cola de } \\
\text { Front Office. } \\
\text { - [992; } 1,479] \text { minutos } \\
\text { para el tiempo de } \\
\text { espera en la cola de } \\
\text { Back Office. } \\
\text { - [28.7; } 47.0] \text { por ciento } \\
\text { de los reclamos } \\
\text { exceden el tiempo } \\
\text { objetivo. } \\
\text { - [24.5; } 42.5] \text { por ciento } \\
\text { de las variaciones } \\
\text { exceden el tiempo } \\
\text { objetivo. }\end{array}$ \\
\hline
\end{tabular}

Fuente: Elaboración propia

\section{PROPUESTAS DE SOLUCIÓN}

Se evaluaron tres propuestas de solución que se resumen en la Tabla $\mathrm{N} .{ }^{\circ} 10$.

Como se aprecia en la Tabla 10, los porcentajes de reclamos y variaciones que exceden los tiempos objetivos son bajos, sobre todo en las propuestas 1 y 3.

A continuación se hará una evaluación económica de la situación actual y de las tres propuestas. El sueldo de un ejecutivo de atención al cliente es S/. 2,500 mensuales.

Evaluación económica de la situación actual

- El personal empleado está compuesto por 45 ejecutivos del grupo Front Office Corporativo y ocho ejecutivos del grupo Back Office Corporativo que ayudan en labores de Back Office durante $45.5 \%$ del tiempo (tres horas y treinta y ocho minutos). 
Tabla N. ${ }^{\circ}$ 10: Resumen de las propuestas de solución para atención de reclamos y variaciones.

\begin{tabular}{|c|c|c|}
\hline $\begin{array}{l}\text { Número de } \\
\text { propuesta }\end{array}$ & Descripción & Intervalos de confianza \\
\hline 1 & $\begin{array}{l}\text { Área de Front Office } \\
\text { - } 45 \text { empleados de Front Office Corporativo } \\
\text { atienden casos de reclamo y variación } \\
\text { durante seis horas diariamente. } \\
\text { Área de Back Office } \\
\text { - } 4 \text { empleados de Back Office Corporativo } \\
\text { resuelven casos de variación durante ocho } \\
\text { horas diariamente. } \\
\text { - } 45 \text { empleados de Front Office Corporativo } \\
\text { resuelven casos de reclamo y variación } \\
\text { durante una hora y cuarenta y cinco minutos } \\
\text { diariamente. }\end{array}$ & $\begin{array}{l}\text { - }[464 ; 497] \text { minutos para el tiempo total de atención } \\
\text { de reclamos. } \\
\text { - }[345 ; 377] \text { minutos para el tiempo total de atención } \\
\text { de variaciones. } \\
\text { - }[0.669 ; 0.680] \text { minutos para el tiempo de espera en } \\
\text { la cola de Front Office. } \\
\text { - }[341 ; 372] \text { minutos para el tiempo de espera en la } \\
\text { cola de Back Office. } \\
\text { - }[0.00 ; 0.32] \text { por ciento de los reclamos exceden el } \\
\text { tiempo objetivo. } \\
\text { - }[0 ; 0.18] \text { por ciento de las variaciones exceden el } \\
\text { tiempo objetivo. }\end{array}$ \\
\hline 2 & $\begin{array}{l}\text { Área de Front Office } \\
\text { - } \quad \begin{array}{l}45 \text { empleados de Front Office Corporativo } \\
\text { atienden casos de reclamo y variación } \\
\text { durante seis horas diariamente. }\end{array} \\
\text { - } \quad \begin{array}{l}\text { Corporativo atienden casos de reclamo y } \\
\text { variación durante seis horas diariamente. }\end{array} \\
\text { Área de Back Office } \\
\text { - } \quad 45 \text { empleados de Front Office Corporativo } \\
\text { resuelven casos de reclamo y variación } \\
\text { durante una hora y cuarenta y cinco minutos } \\
\text { diariamente. } \\
\text { - } 3 \text { nuevos empleados de Front Office } \\
\text { Corporativo resuelven casos de variación } \\
\text { durante dos horas diariamente. } \\
\text { - empleados de Back Office Corporativo } \\
\text { resuelven casos de variación durante ocho } \\
\text { horas diariamente. }\end{array}$ & $\begin{array}{l}\text { - }[575 ; 645] \text { minutos para el tiempo total de } \\
\text { atención de reclamos. } \\
\text { - }[455 ; 525] \text { minutos para el tiempo total de } \\
\text { atención de variaciones. } \\
\text { - }[0.621 ; 0.630] \text { minutos para el tiempo de espera } \\
\text { en la cola de Front Office. } \\
\text { - }[447 ; 516] \text { minutos para el tiempo de espera en la } \\
\text { cola de Back Office. } \\
\text { - }[0.63 ; 4.00] \text { por ciento de los reclamos exceden el } \\
\text { tiempo objetivo. } \\
\text { [0.18; } 2.85] \text { por ciento de las variaciones exceden } \\
\text { el tiempo objetivo. }\end{array}$ \\
\hline 3 & $\begin{array}{l}\text { Área de Front Office } \\
\text { - } \quad \begin{array}{l}43 \text { empleados de Front Office Corporativo } \\
\text { atienden casos de reclamo y variación } \\
\text { durante seis horas diariamente. }\end{array} \\
\begin{array}{ll}\text { Área de Back Office } \\
\text { - }\end{array} \\
\begin{array}{l}\text { resuelven casos de reclamo y variación } \\
\text { durante una hora y cuarenta y cinco }\end{array} \\
\text { minutos diariamente. } \\
2 \text { empleados de Front Office Corporativo } \\
\text { resuelven casos de variación durante ocho } \\
\text { horas diariamente. } \\
\text { 3 empleados de Back Office Corporativo } \\
\text { resuelven casos de variación durante ocho } \\
\text { horas diariamente. }\end{array}$ & $\begin{array}{l}\text { - }[348 ; 365] \text { minutos para el tiempo total de } \\
\text { atención de reclamos. } \\
\text { - }[226 ; 243] \text { minutos para el tiempo total de } \\
\text { atención de variaciones. } \\
\text { - }[0,679 ; 0.685] \text { minutos para el tiempo de espera } \\
\text { en la cola de Front Office. } \\
\text { - }[233 ; 236] \text { minutos para el tiempo de espera en la } \\
\text { cola de Back Office. } \\
\text { - }[0.0 ; 0.0] \text { por ciento de los reclamos exceden el } \\
\text { tiempo objetivo. } \\
\text { [0.0;0.0] por ciento de las variaciones exceden el } \\
\text { tiempo objetivo. }\end{array}$ \\
\hline
\end{tabular}


- El porcentaje promedio de utilización de cada uno de los 45 ejecutivos del grupo Front Office Corporativo dedicados a atender y resolver casos de reclamo y variación es $71.44 \%$.

- El porcentaje promedio de utilización de cada uno de los 8 ejecutivos del grupo Back Office Corporativo dedicados a resolver casos de variación es $85.06 \%$.

- Entonces, el costo mensual es: $45 \mathrm{x}$ $(2,500 / 0.7144)+8 \times(2,500 / 0.8506)^{*} 0.455=\mathrm{S} /$. $168,173.34$.

\section{Evaluación económica de la propuesta 1}

- El personal empleado está compuesto por 45 ejecutivos del grupo Front Office Corporativo y cuatro ejecutivos del grupo Back Office Corporativo ayudan en labores de Back Office durante $100 \%$ del tiempo (ocho horas).

- El porcentaje promedio de utilización de cada uno de los 45 ejecutivos del grupo Front Office Corporativo dedicados a atender y resolver casos de reclamo y variación es $70.73 \%$.

- El porcentaje promedio de utilización de cada uno de los 4 ejecutivos del grupo Back Office Corporativo dedicados a resolver casos de variación es $98.66 \%$.

- Entonces, el costo mensual es: $45 \mathrm{x}$ $(2,500 / 0.7144)+4 \times(2,500 / 0.9866)=\mathrm{S} /$. $169,191.38$.

\section{Evaluación económica de la propuesta 2}

- El personal empleado está compuesto por 45 ejecutivos del grupo Front Office Corporativo, 3 nuevos ejecutivos del grupo Front Office Corporativo y 3 ejecutivos del grupo Back Office Corporativo que ayudan en labores de Back Office durante $100 \%$ del tiempo (ocho horas).

- El porcentaje promedio de utilización de cada uno de los 45 ejecutivos del grupo Front Office Corporativo dedicados a atender y resolver casos de reclamo y variación es $68.27 \%$.

- El porcentaje promedio de utilización de cada uno de los 3 nuevos ejecutivos del grupo Front Office Corporativo dedicados a atender y resolver casos de reclamo y variación es $68.91 \%$.

- El porcentaje promedio de utilización de cada uno de los 3 ejecutivos del grupo Back Office Corporativo dedicados a resolver casos de variación es $99.41 \%$.
- Entonces, el costo mensual es: $45 \mathrm{x}$ $(2,500 / 0.6827)+3 x(2,500 / 0.6891)+3 x$ $(2,500 / 0.9941)=S / .183,215.15$.

Evaluación económica de la propuesta 3

- El personal empleado está compuesto por 43 ejecutivos del grupo Front Office Corporativo, dos ejecutivos del grupo Front Office Corporativo dedicados sólo a labores de Back Office y tres ejecutivos del grupo Back Office Corporativo que ayudan en labores de Back Office durante $100 \%$ del tiempo (ocho horas).

- El porcentaje promedio de utilización de cada uno de los 43 ejecutivos del grupo Front Office Corporativo dedicados a atender y resolver casos de reclamo y variación es $72.08 \%$.

- El porcentaje promedio de utilización de cada uno de los 2 ejecutivos del grupo Front Office Corporativo dedicados a resolver casos de variación es $94.83 \%$.

- El porcentaje promedio de utilización de cada uno de los 3 ejecutivos del grupo Back Office Corporativo dedicados a resolver casos de variación es $97.52 \%$.

- Entonces, el costo mensual es: $43 \times$ $(2,500 / 0.7208)+2 \times(2,500 / 0.9483)+3 x$ $(2,500 / 0.9752)=S / .162,103.17$.

Por lo tanto, la propuesta 3 es la que se recomienda, ya que cumple con no exceder los tiempos objetivos para los casos de reclamos y variaciones, y además es la alternativa menos costosa tomando en cuenta la utilización de los recursos humanos.

\section{CONCLUSIONES}

Las principales conclusiones de la investigación son:

- El porcentaje de reclamos y variaciones que son solucionadas fuera del tiempo objetivo es bastante elevado, entre $28.7 \%$ y $47.0 \%$ en el caso de reclamos y entre $24.5 \%$ y $42.5 \%$ en el caso de variaciones. Por ello, el sistema actual es ineficiente y esto repercute negativamente en la satisfacción de los clientes, pues definitivamente es importante para ellos que su solicitud sea atendida a la brevedad posible, más aún si se trata de reclamos. Por ello se deben realizar mejoras para que se incremente la satisfacción del cliente.

- La primera propuesta recomienda que cuatro ejecutivos de Back Office Corporativo ayuden a resolver casos de variación durante ochos ho- 
ras. Esta propuesta permite resolver todos los reclamos y variaciones sin exceder los tiempos objetivos.

- La tercera propuesta recomienda que tres ejecutivos de Back Office Corporativo ayuden a resolver casos de variación durante ochos horas. Además recomienda que dos ejecutivos de Front Office Corporativo se dediquen a resolver casos también durante ocho horas. Esta propuesta permite resolver todos los reclamos y variaciones sin exceder los tiempos objetivos.

- La evaluación económica indica que la propuesta menos costosa es la tercera. El costo mensual es S/. 162,103.17.

- La propuesta más conveniente es la tercera debido a que los indicadores de atención de reclamos y variaciones son mejores que las demás propuestas, incluyendo a la situación actual, y además tiene los menores costos asociados a recursos humanos.

\section{REFERENCIAS BIBLIOGRÁFICAS}

[1] Brousset M.O. (2007), Análisis y Mejora de los Procesos de Atención al Cliente en un Centro de Llamadas de una Empresa de Telecomunicaciones. Tesis (Ingeniera Industrial), Pontificia Universidad Católica del Perú, Facultad de Ciencias e Ingeniería, Lima, Perú.

[2] Fitzsimmons, J. A. y Fitzsimmons, M. J. (2008). Service Management: Operations, Strategy and Information Technology. 6ta. ed. McGraw-Hill/ Irwin.

[3] Kelton, W.D.; Sadowski, R.P. And Sturrock, D.T. (2008), Simulación con Software Arena. Cuarta ed. McGraw Hill.

[4] Torres, P. (2010), Simulación de Sistemas con el software Arena. Fondo Editorial, Universidad de Lima. 